\title{
Importância do serviço especializado no tratamento das hérnias da parede abdominal de alta complexidade
}

\section{Role of specialized center in management of highly complex abdominal wall hernias}

Carlos Augusto de Mattos Donadelli

"Se nenhuma outra área fosse oferecida a um Cirurgião para sua atividade a não ser a Herniotomia, ainda assim valeria a pena ter sido um Cirurgião e devotar uma vida inteira a este serviço” (Halsted, 1892) ${ }^{1}$

Nos primórdios da nossa espécie, a capacidade de adotar a postura ereta trouxe à humanidade a possibilidade de explorar melhor seu ambiente e desenvolver grandes progressos em um curto espaço de tempo. A maior parte do mundo repleto de maravilhas tecnológicas em que vivemos deve-se a este feito extraordinário. Carregaremos ainda por alguns milhões de anos o fardo das conseqüências físicas desse recente salto evolutivo, como os transtornos da coluna vertebral, das articulações do joelho, quadris, tornozelos, as doenças ligadas aos esfíncteres pélvicos e as hérnias da parede abdominal que compreendem as hérnias inguinais, umbilicais, ventrais, incisionais e todos os defeitos mioaponeuróticos no território da parede abdomino-pélvica. Um baixo custo, tendo em vista a contrapartida, mas uma conta a pagar por incontáveis gerações.
A relação da medicina, sobretudo da cirurgia com as hérnias da parede abdominal é muito antiga, repleta de grandes feitos científicos e avanços objetivos, mas também carregada de aspectos emocionais e subjetividade. De um lado temos as técnicas revolucionárias em cada período da história, como a de Bassini (1889) $)^{2}$, McVay (1942) $)^{3}$, Shouldice $(1945)^{4}$, a contribuição brasileira do Prof. Alcino Lázaro $(1971)^{5}$ e tantos outros para a hérnia ventral, as mais recentes conquistas técnicas após à introdução das próteses como Stoppa $(1984)^{6}$, Litchenstein $(1986)^{7}$ e Gilbert $(1999)^{8}$, as modernas técnicas videolaparoscópicas como abordagem transperitonial (TAPP) ${ }^{9}$, extraperitonial (TEP) ${ }^{10}$ na hérnia inguinal, ou ainda a aplicação da via laparoscópica no tratamento das hérnias ventrais ${ }^{11}$; por fim os avanços mais recentes como a cirurgia robótica, o desenvolvimento de materiais sintéticos absorvíveis e menos imunogênicos, grampeadores e cola de fibrina direcionando o tratamento das hérnias para o futuro cada vez menos invasivo, mais eficaz e com menores sequelas ${ }^{12}$. Pelo lado emocional, observamos a dor e o desconforto que pode somente ser descrito pelas pessoas portadoras desta
Pós-graduando (doutorado) do Departamento de Cirurgia e Anatomia da Faculdade de Medicina de Ribeirão Preto da Universidade de São Paulo (FMRP-USP)

Médico assistente da disciplina de Cirurgia Digestiva do Hospital das Clínicas da FMRP/USP
Correspondencia: Departamento de Cirurgia e Anatomia - HCRP Av. dos Bandeirantes, 3900. Campus Universitário, Monte Alegre 14049-900 - Ribeirão Preto - SP e-mail: camdonadelli@ hotmail.com / camdonadelli@usp.br Aprovado para publicação em 22/12/2010 
doença, a expectativa do residente de cirurgia tem nas cirurgias de hérnia os primeiros passos de sua formação, a opinião dos cirurgiões experientes na preferência por esta ou aquela técnica, permitindo-se omitir, acrescentar e modificar os passos técnicos com base apenas na própria observação não sistematizada e na análise sentimental da própria casuística. Dada a grande frequiência dessas doenças, muitos profissionais com um razoável número de casos tratados consideram-se autoridades no assunto. Será que há lugar para maior especialização?

Mais de 20 milhões de herniorrafias são realizadas por ano no mundo. Os números variam entre 100 e 300 procedimentos por 100000 habitantes nos diversos países ${ }^{13}$. Um grande impacto econômico decorre da incapacidade laboral e dos custos produzidos pela doença herniária simples ou complicada. Neste inicio de século XXI, com todos os avanços neste campo, as recidivas ainda estão presentes em até 32\% das hérnias incisionais ${ }^{14}$, problemas como dor crônica atingem cerca de $10 \%$ dos pacientes em pós-operatório de hérnia inguinal ${ }^{15}$, inúmeras alterações funcionais, complicações graves e mortalidade não desprezível decorre do tratamento cirúrgico ou da sua falta. Certamente estamos à frente de nossos colegas de dois séculos atrás, mas é necessário refletir se alguns números são simplesmente aceitáveis ou não receberam a devida atenção.

A sub-especialização das áreas cirúrgicas é um processo consolidado. Dentro das divisões clássicas surgiram novas áreas. Na cirurgia digestiva, por exemplo, há divisões dedicadas à cirurgia do esôfago, estômago, fígado, vias biliares, pâncreas, cólon, cada segmento contando com muitos adeptos. Em algum lugar cada vez menor entre a tradicional cirurgia geral, a cirurgia plástica, a cirurgia digestiva e outras especialidades encontra-se o cuidado com a parede abdominal. Com frequiência esta área vem sendo relegada ao segundo plano em grandes serviços especializados. Em diversos hospitais, a correção das hérnias é relegada a médicos residentes do primeiro ano sob orientação de seus pares do segundo ou terceiro. Não há dúvidas acerca da importância das herniorrafias na formação das próximas gerações de cirurgiões, mas os números mostram que é preciso maior comprometimento das equipes com essas doenças tão freqüentes.

Além do contexto individual ou institucional, dada a alta prevalência da doença herniária, o assunto precisa ser visto com seriedade pelas autoridades de saúde pública. Na Divisão Regional de Saúde de Ribeirão Preto (DRS 13), observamos o impacto positivo da recente implantação do Hospital Estadual. Existe uma tendência a aumento progressivo da quantidade de herniorrafias realizadas neste serviço ao longo dos quatro anos de existência da instituição (Figura 1).

O Hospital Estadual de Ribeirão Preto recebe pacientes de todos os 26 municípios da DRS ${ }^{13}$. Nesta instituição são feitas cirurgias de médio porte em regime ambulatorial ou com curto período de internação. Este modo de funcionamento permite rápida resolu-

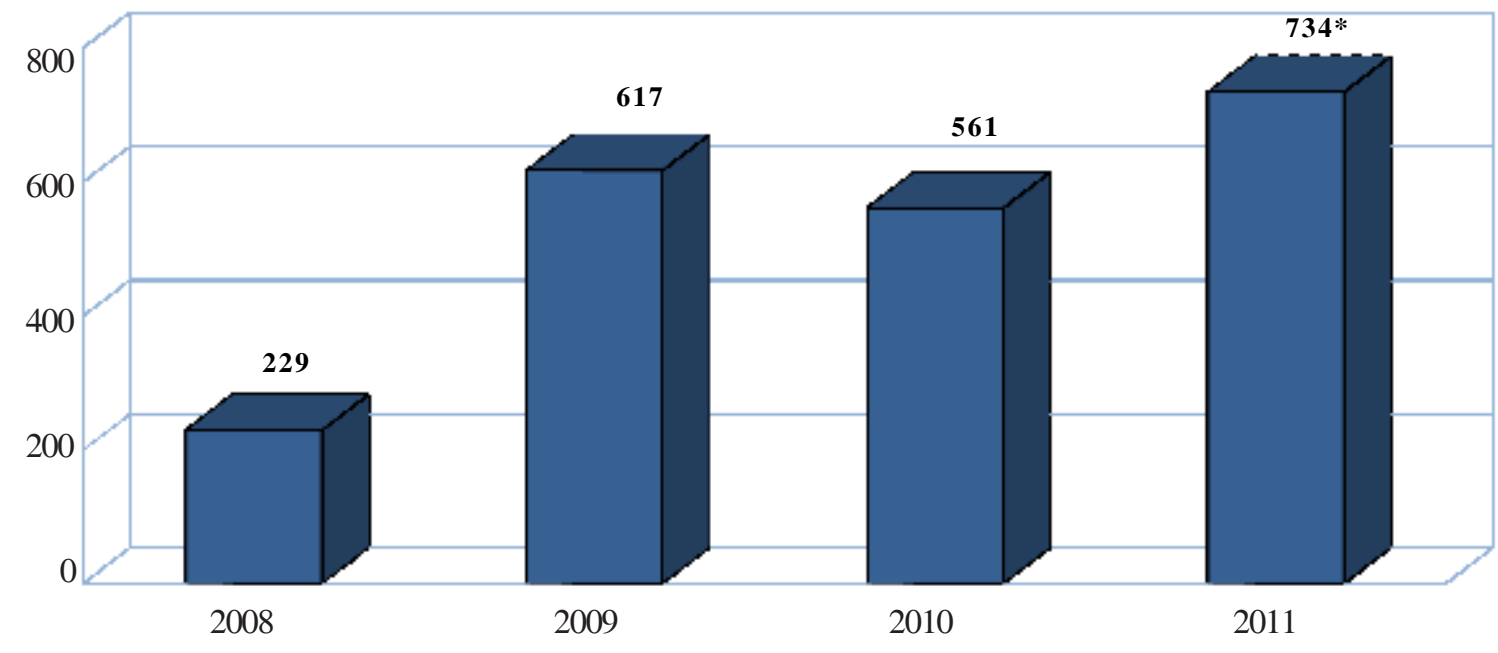

Figura 1. Número de herniorrafias realizadas Hospital Estadual de Ribeirão Preto no período de 2008 a 2011.

*dados parciais, referentes de janeiro a novembro de 2011. 
ção dos problemas e grande impacto epidemiológico. Assim, muitas complicações da doença herniária foram evitadas, inúmeros casos foram tratados em fases iniciais com reabilitação completa, salvando preciosos recursos de saúde e de outras esferas econômicas, por tratar-se de doença de grande impacto ocupacional. No entanto é preciso lembrar que o Hospital Estadual de Ribeirão Preto é uma instituição de nível secundário planejada para oferecer tratamento de média complexidade. Este serviço não tem capacidade para tratar hérnias de grande volume, hérnias complexas, muitas vezes com perda de domicílio das vísceras abdominais, além de portadores com múltiplas comorbidezes, necessidade de suporte ventilatório, cardiovascular, dentre outras. Assim, do ponto de vista operacional do sistema de saúde podemos dividir a doença herniária em dois grupos: média e alta complexidade. No primeiro, como visto há mais acesso ao tratamento em nossa região. Vejamos o que vem ocorrendo com o segundo grupo.

A disciplina de Cirurgia Geral do Hospital das Clínicas de Ribeirão Preto é o maior serviço terciário da região para tratamento de hérnias e outras afecções da parede abdominal. Nos últimos anos observamos redução progressiva da equipe, leitos e horários cirúrgicos eletivos para outras dessa disciplina tendo como consequiência grande queda no volume cirúrgico (Figura 2).

Durante os anos de 2008 e 2009 houve suspensão da triagem de novos casos nessa disciplina, devido à grande quantidade de pacientes já avaliados que aguardavam tratamento cirúrgico. No entanto, pacientes já matriculados no hospital, oriundos de outras clínicas continuaram a receber indicação. Em 2010, com a retomada das atividades houve um aumento ainda maior da demanda, superando em muito a quantidade de casos tratados nos últimos 2 anos (Figura 3).

Atualmente, há 224 pacientes aguardando cirurgia para tratamento de hérnia da parede abdominal no HCRP (dados de 30/11/2011), sendo que a quase totalidade excede os critérios para tratamento no Hospital Estadual ou outras instituições secundárias da DRS. Um grande problema para a instituição e para os gestores de saúde pública.

A longo prazo é preciso também estimular o interesse das novas gerações de cirurgiões para a doença herniária, um campo muito vasto da literatura médica, mas no qual ainda existe uma série de questões a responder. O tratamento das hérnias da parede abdominal de grande porte requer técnica cirúrgica apurada, conhecimentos médicos variados, domínio da fisiologia, imunologia, processo de cicatrização e outras ciências básicas; rápida capacidade de adaptação às incorporações técnicas, mas acima de tudo um grau de comprometimento que só pode ser atingido por cirurgiões dedicados a essa área. Também é essencial a interação com outros profissionais médicos e não médicos, pois tratando-se de uma doença de múltiplos aspectos (condições sociais e ocupacionais, mecânica ventilatória, necessidade de perda ponderal, aspectos psicológicos relacionados à dor crônica, reabilitação, anestesia especializada, cuidados intensivos,

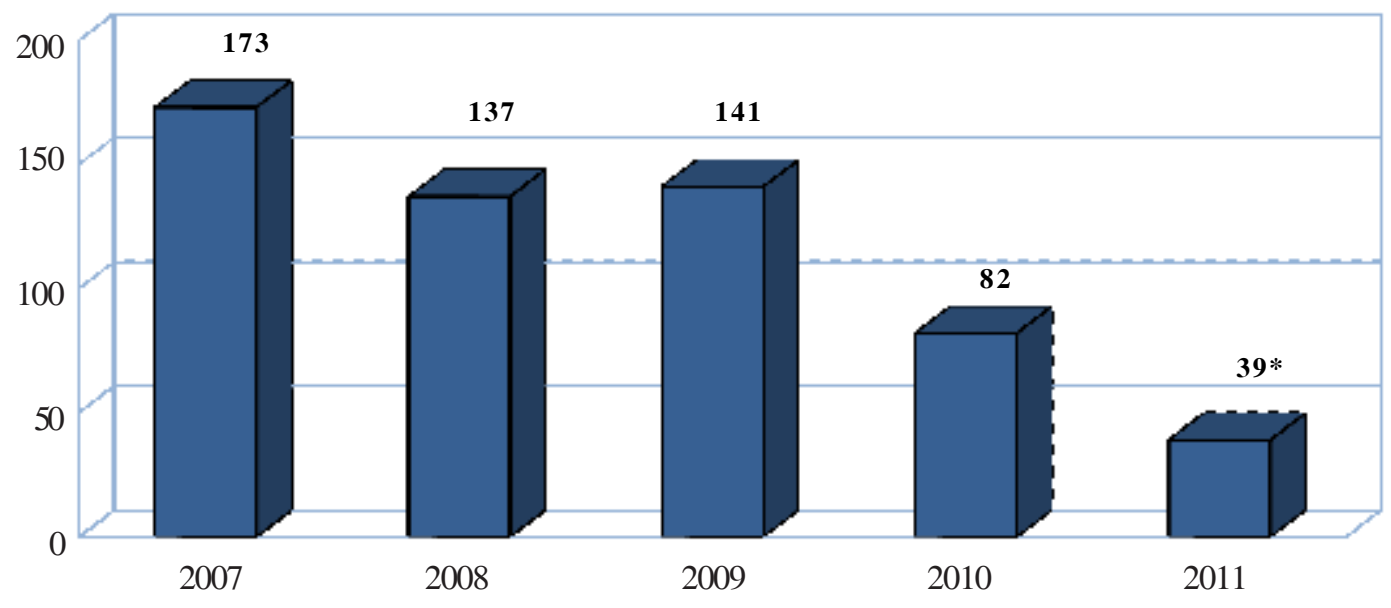

Figura 2. Número de herniorrafias realizadas pelo Serviço de Cirurgia Geral do HCFMRP/USP no período de 2007 a 2011 (até 28/11/2011). *dados parciais, referentes de janeiro a novembro de 2011. 


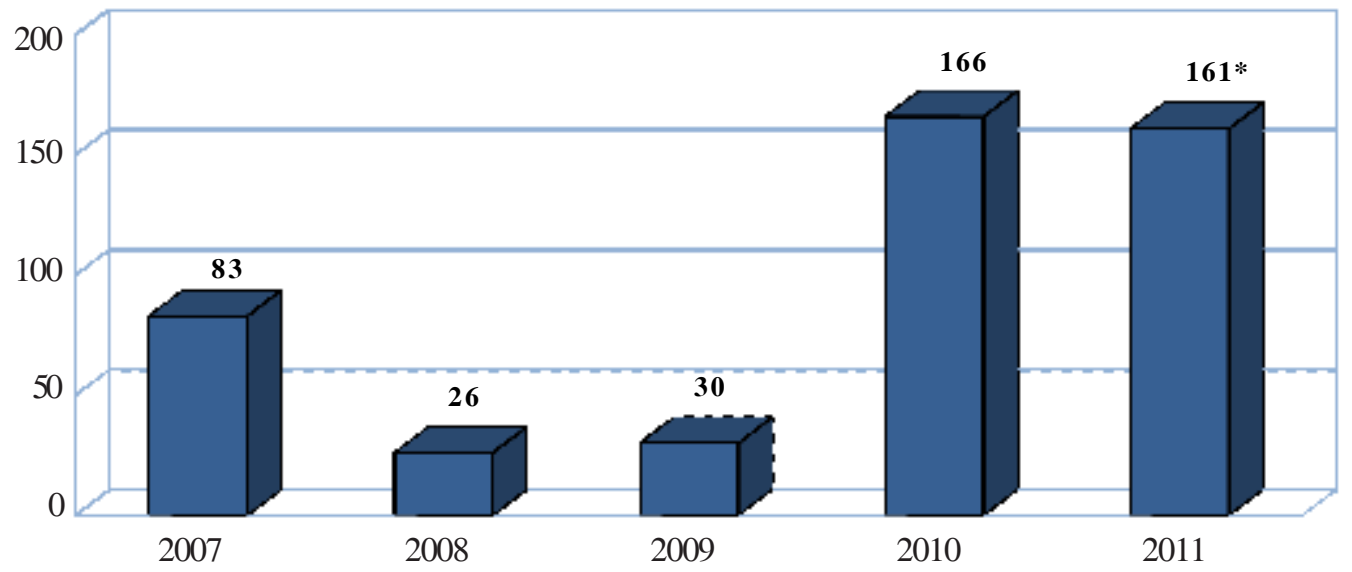

Figura 3. Número de pacientes incluídos na lista de espera para tratamento cirúrgico de hérnias da parede abdominal no HCFMRP/USP no período de 2007 a 2011. *dados parciais, referentes de janeiro a novembro de 2011.

etc), deve receber atenção multidisciplinar. A formação de uma equipe especializada em defeitos da parede abdominal, além de solucionar o problema de milhares de pacientes é capaz de contribuir para melhorar a formação e o ensino médico, abrindo novas portas para o desenvolvimento técnico e científico da instituição.

"Nenhuma doença do corpo humano, pertencente aos domínios do cirurgião, requer em seu tratamento uma melhor combinação de acurácia, conhecimento anatomico e habilidade cirúrgica que a hérnia em todas as suas variedades". (Astley Cooper, 1804).

\section{Referências}

1- Halsted WS. The Radical Cure of Inguinal Hernia in the Male. Ann Surg 1893; 17(5):542-56.

2- Sachs $M$, Damm M, Encke A. Historical evolution of inguinal hérnia repair. Bassini E. World J Surg 1997; 21(2): 218-23.

3- McVay CB, Anson, BJ. A fundamental error in current methods of inguinal herniorrhaphy. Surg Gynecol Obstet 1942; 74:746,

4- Shearburn EW, Myers RN. Shouldice repair for inguinal hernia. Surgery 1969; 66(2): 450-59.
5- Lázaro-da-Silva A. Plástica com o saco herniário na correção cirúrgica das hérnias incisionais. O Hospital 1971;79:12334.

6- Stoppa RE, Rives JL, Warlaumont CR, Palot JP, Verhaeghe PJ, Delattre JF. The use of Dacron in the repair of hernias of the groin. Surg Clin North Am 1984; 64:269-85.

7- Lichtenstein IL, Shulman AG. Ambulatory outpatient hernia surgery. Including a new concept, introducing tension-free repair. Int Surg 1986; 71:1-4.

8- Gilbert AI, Graham MF, Voigt WJ. A bilayer patch device for inguinal hernia repair. Hernia 1999; 3(3):161-66.

9- Bittner R, Schmedt CG, Schwarz J, Kraft K, Leibl BJ. Laparoscopic transperitoneal procedure for routine repair of groin hernia. Br J Surg 2002; 89:1062-66.

10- Dulucq JL. Treatment of inguinal hernia by insertion of a subperitoneal patch under preperitoneoscopy. Chirurgie 1992; 118:83-85

11- LeBlanc KA, Booth WV.Laparoscopic repair of incisional abdominal hernias using expanded polytetrafluoroethylene: preliminary findings. Surg Laparosc Endosc 1993; 3 (1):3941.

12- Read RC. Herniology: past, present and future. Hernia 2009;13: 577-80.

13- Kingsnorth A, LeBlanc K. Hernias: inguinal and incisional. Lancet 2003; 362:1561-71.

14- Park AE, Roth JS, Kavic SM. Abdominal Wall Hernia. Current Problems in Surgery 2006; 43(5): 326-75.

15- Hakeem A, Shanmugam V. Inguinodinia following Litchenstein tension-free hernia repair: a review. World J Gastroenterol 2011; 17(14):1791-96. 